\title{
Cooperação em saúde em fronteiras internacionais: a busca da igualdade em saúde
}

Health cooperation in cross borders - the pursuit of equality in health

\section{Carla Gabriela Cavini Bontempo}

Administradora. Doutoranda no Programa de Pós-Graduação em Serviço Social da Universidade Federal de Santa Catarina. Florianópolis, Brasil.

\section{Vera Maria Ribeiro Nogueira}

Assistente Social. Doutora em Enfermagem. Programa de Política Social na Universidade Católica de Pelotas e Pós-Graduação em Serviço Social na Universidade Federal de Santa Catarina. Florianópolis, Brasil.

\section{Roser Perez Gimenez}

Enfermeira. Doutora em Sociologia. Universidade de Terrassa, Espanha.

Resumo: Este informe contêm os resultados do estudo sobre a cooperação entre sistemas de saúde apresentando quatro experiências consideradas como bem sucedidas de cooperação/integração/ harmonização dos sistemas e/ou serviços de saúde em linhas de fronteira. Os dados foram obtidos através de entrevistas com gestores e profissionais de saúde das cidades de Badajoz, Puigcerdã - Espanha e Foz do Iguaçu, Dionísio Cerqueira e Santana do Livramento - Brasil. Os principais achados se referem a: os antecedentes das iniciativas são similares, pois com exceção da tríplice fronteira, as demais têm como foco mobilizador as necessidades sociais em saúde, sendo determinante a dificuldade de acesso dos nacionais aos recursos de atenção básica, principalmente, de média e alta complexidade; destacam-se, em todas as experiências, o protagonismo dos profissionais e gestores dos sistemas locais de saúde na articulação de estratégias para fomentar a cooperação; outro aspecto que favoreceu os processos de cooperação estudados foi serem iniciativas que partem de um ente público, tanto na prestação como financiamento dos serviços. Os obstáculos se situam na excessiva formalização para atuação interfronteiras. Concluindo pode-se alertar, nas fronteiras brasileiras, para o papel desempenhado pelos comitês de fronteira e comitês de saúde para mobilizar as forças políticas locais em direção à cooperação em saúde. Há, no entanto, a necessidade de ampliar o espectro político para além do partidário e obter uma expertise para manejo dos recursos governamentais disponíveis.

Palavras-chave: Cooperação internacional em saúde; saúde em fronteiras; política de saúde.

Key-words: Cross-border health; health cooperation; health care; cross-border cooperation; patient rights 
Este trabalho sintetiza as ações realizadas no âmbito da pesquisa Ampliando o direito à saúde - experiências de cooperação entre sistemas e/ou serviços de saúde em linha de fronteira, desenvolvido entre agosto de 2009 e agosto de 2011. Contêm a descrição das atividades e os principais resultados obtidos quanto aos objetivos da pesquisa. Aborda o tema de cooperação entre sistemas de saúde ou entre ações e serviços de saúde apresentando especialmente as experiências consideradas como bem sucedidas de cooperação/integração/harmonização dos sistemas e/ou serviços de saúde em linhas de fronteira, tendo como objetivos: conhecer a história e a trajetória recente das experiências de cooperação entre serviços e/ou sistemas de saúde no Mercado Comum do Sul (MERCOSUL) e União Europeia; identificar e analisar o processo de implementação evidenciando os atores políticos envolvidos e os interesses presentes em seu desenvolvimento; conhecer o nível de institucionalização das experiências de cooperação transfronteiriça dos serviços e/ou sistemas de saúde; identificar as formas e procedimentos jurídicos, administrativos e financeiros relacionados com a harmonização dos sistemas; estabelecer os nexos entre as boas práticas e os elementos determinantes de sua configuração e institucionalidade.

O tema da atenção à saúde em áreas fronteiriças aparece timidamente no debate na primeira metade da década de 2000, essencialmente orientado pela Organização Mundial de Saúde (Astorga, 2004). A Constituição brasileira de 1988 define como faixa de fronteira a área compreendida dentro de cento e cinquenta quilômetros perpendiculares à linha do limite do território brasileiro. Atualmente se estabeleceu o uso do termo linha de fronteira que seria o território municipal que compõe a divisa nacional. Cidades-gêmeas são as que confrontam com outras de outro país, sem limites físicos marcantes (Brasil, 2006). Hoje se identifica maior número de estudos sobre a questão, notadamente em regiões onde os conflitos sociais são mais candentes, ou apresentam maior visibilidade, como o limite entre México e Estados Unidos.

$\mathrm{Na}$ América Latina, somente nos últimos quatro anos houve um relativo adensamento da produção e, no mais das vezes, voltado para aspectos epidemiológicos e de vigilância sanitária. Sobre a situação dos sistemas de saúde, iniqüidade em saúde, situações perversas e evitáveis vivenciadas na região fronteiriça, pouco se tem publicado. Destacam-se, entre outros, os estudos de 
Astorga (2004), Silva (2006); Guimarães e Giovanella (2005a, 2005b,); Nogueira e Dal Prá (2006, 2007), Nogueira (2006, 2007, 2008), Giovanella et all (2006); Gallo (2004), Dain (2004), Simionatto e Nogueira (2004).

Quanto às iniciativas de cooperação transfronteiriça, Guimarães e Giovanella (2005), Giovanella et all (2006) e Guimarães e Queiroz (2007) resgatam iniciativas de cooperação entre sistemas de saúde, sendo duas experiências na fronteira MERCOSUL e uma terceira na euro-região da Extremadura - Espanha e Alentejo Portugal. Nogueira e Pérez (2008) descrevem estudo exploratório realizado na fronteira Espanha-França.

A busca de artigos sobre harmonização/cooperação e integração de sistemas em fronteiras nas bases bibliográficas (Lilacs, Scielo, Cochrane Library, MedLine, Dialnet e Redalyc) foi infrutífera. Confirma-se assim, que

\begin{abstract}
Não existem, tão pouco, estudos sobre a convergência de sistemas de saúde, nem mesmo no âmbito do Mercosul. Tal insuficiência de informações tem correspondência na compreensão e na construção de alternativas. Isso implica em que as soluções propostas não sejam claras e objetivas e esteja limitada, na maioria das vezes, ao aspecto financeiro e à formação de comitês para discussão. Embora esse dois aspectos sejam relevantes têm que estar referidos a objetivos claros, metas e critérios de viabilidade e sustentabilidade. O problema assume dimensões de relações internacionais e deve ser tratado com status equivalente na agenda da saúde dos Estados-Parte (Guimarães e Giovanella, 2005, p. 11).
\end{abstract}

A situação dos sistemas de saúde em região de fronteira entrou na agenda do debate internacional mais pelas consequências do reordenamento econômico, devido à crise dos anos 1990/2000, e menos pela insatisfação e demanda dos gestores e da população residente na área. Há, entretanto, o reconhecimento, pelos gestores, do aumento dos fluxos e intercâmbios de pessoas, de serviços e de produtos decorrentes dos processos de integração regional, repercutindo diretamente nos indicadores sanitários e demográficos das cidades fronteiriças, que se tornam corredores econômicos (Arboleda-Florez et al, 1999, apud Giovanella, 2004).

O plano metodológico orientou-se por duas categorias - historicidade e institucionalidade e os dados obtidos através de entrevistas com gestores e profissionais de saúde das cidades de Badajoz, Puigcerdã- Espanha e Foz do Iguaçu, Dionísio Cerqueira e Santana do Livramento-Brasil. Em caso de indicação dos gestores ou profissionais também se entrevistou informantes qualificados nas cidades de Rivera e Bernardo de Yrigoyen. Privilegiou-se, ainda a analise documental 
(relatos, atas de reuniões e pactos formalizados) obtidos junto aos gestores. O estudo articulou pesquisadores de distintas instituições acadêmicas do sul da América Latina, parceiras nos projetos desenvolvidos anteriormente através da cooperação de pesquisadores das: Universidade de La República - Uruguai, Universidade Nacional de Assunção - Paraguai, Universidade Nacional de Misiones- Argentina e Faculdade União das Américas-Foz do Iguaçu, Universidade Federal de Santa Catarina, Escola Nacional de Saúde Pública da FIOCRUZ, o Grupo Seminário de Avaliação de Políticas Sociais (SAPS), da Universidade Autônoma de Barcelona/Escola Creu Roja de Enfermeria da Catalunha, Espanha, e, mais recentemente, incluiu uma pesquisadora do Instituto Social do Mercosul. Participaram pelo Brasil, pesquisadores das instituições a seguir: UCPEL, UNIJUÍ, UNIAMÉRICA e UFSC.

Os principais resultados indicam que ainda que as particularidades se expressem, um ponto de aproximação encontrado foi o relacionado aos antecedentes das iniciativas de cooperação entre os sistemas de saúde. Com exceção da tríplice fronteira, as demais experiências têm como foco mobilizador as necessidades sociais em saúde, sendo determinante a dificuldade de acesso dos nacionais aos recursos de atenção básica, em alguns casos e principalmente de média e alta complexidade. Esta dificuldade de acesso diz respeito a aspectos objetivos como a distância geográfica e os efeitos climáticos, ou seja, o atendimento aos nacionais se impossibilita devido à estes aspectos, sendo que do outro lado da fronteira, os recursos estão disponíveis.

Nas experiências analisadas destacam-se duas esferas estruturais determinantes das desigualdades - a estatal e a relacional, e em segundo plano a esfera mercantil. No plano estatal identifica-se uma grande distinção entre as experiências situadas no âmbito do Mercosul e da União Europeia. No território europeu o papel e a função do Estado, em qualquer dos níveis de abrangência nacional/regional e local - possui uma capacidade intrínseca de governança e responsabilidade administrativa, evidente no financiamento, controle e acompanhamento das ações realizadas, as quais tem a chancela dos governos respectivos, firmando-se na legislação e procedimentos administrativos em vigor na União Européia. Na linha da fronteira do Mercosul, se identifica uma alternância de ordenamentos programáticos e intencionalidades políticas sedimentando-se mais em torno de interesses que não partem propriamente dos gestores locais ou da 
legislação nacional, mas de ordem corporativa profissional. Em um único caso se constata o envolvimento constante e sistemático do gestor local, que é Santana do Livramento/Rivera. Um ponto em comum na esfera estatal é o fato de todas as iniciativas de cooperação entre os sistemas que foram analisadas partiram das instâncias locais e posteriormente influenciando outras instâncias, provocando alterações no campo jurídico-administrativo inclusive em instâncias supranacionais como está sendo verificada na construção do Hospital da Cerdaña em Puigcerdã/Bourg Madame e instalação do equipamento para tomografias de emissão de posítron-ciclotrón (PET-CT).

Há que se dimensionar o fato que as alterações ocorridas quanto às fronteiras físicas na EU, que ao serem abolidas, favoreceu o trânsito de pessoas, conforme afirma um dos entrevistados.

[...] notó sobretodo cuando se quitó la frontera con el tema de la unión europea, porque como antes teníamos horarios para pasar y para cerrar la frontera y tal, pero cuando la frontera se abrió 24 horas... y ya se quitó la policía, ya no distingue entre quien viene, quien va y quien está aquí (MB).

Outro aspecto voltado à esfera estatal, na linha de fronteira Mercosul é a descontinuidade administrativa ocorrida nas alterações das gestões municipais. Esta questão causou forte impacto no desenvolvimento das iniciativas de cooperação em Dionísio Cerqueira/Bernardo Yrigoyen, as quais eram inclusive chanceladas pelo Governo Estadual de Santa Catarina, pois o município argentino não integra a nova organização de fronteira, além de desconsiderar as ações que vinham sendo realizadas pelo Comitê Internacional de Saúde de Fronteira. O atual CIF - um Consórcio Intermunicipal da Fronteira, do qual somente fazem parte os municípios: Dionísio Cerqueira-SC, Barracão e Bom Jesus do Sul-PR do Brasil, não incluiu o município de Bernardo de Yrigoyen, da Argentina

$\mathrm{Na}$ esfera relacional, as iniciativas dos profissionais vinculadas ao campo da saúde têm sido fundamentais no sentido de iniciarem ações cooperativas. Em todas as experiências há o papel motivador, mobilizador e de gestão da cooperação, embora nem todos com os mesmos ideais éticos e políticos embora se encontre, de forma unânime as referências as desigualdades territoriais em saúde. Algumas falas dos profissionais de Santana Livramento/Rivera e de Dionísio Cerqueira/Bernardo de Yrigoyen sinalizam para uma igualdade em termos de direito universal à saúde, abrangendo todos os níveis de atenção. Já no território europeu duas experiências se 
voltam para instalação de hospital e equipamentos de alta tecnologia, não havendo no discurso dos protagonistas referências tanto a direitos universais como os objetivos de coesão, conforme constam do Programa Operativo de Cooperação Transfronteiriça Espanha-Portugal 2007/2013 (D.G. Fondos Comunitarios España/D.G. de Desarrollo Regional Portugal, 2009).

Ainda em relação a este aspecto, as experiências exitosas, consideradas as de Santana Livramento/Rivera, Puigcerdã/Bourg Madame e Badajoz/Elvas contam com gestores bastante comprometidos com as mesmas, os quais estão à frente das mesmas desde o início. Assim, a continuidade da gestão, mais do que sua formalização legal parece permitir que as institucionalidades delas decorrentes se fortaleçam no plano da gestão, administrativo e financeiro.

Ainda que as particularidades se expressem, um ponto de aproximação encontrado foi o relacionado aos antecedentes das iniciativas de cooperação entre os sistemas de saúde. Com exceção da tríplice fronteira, as demais têm como foco mobilizador as necessidades sociais em saúde, sendo determinante a dificuldade de acesso dos nacionais aos recursos de atenção básica, em alguns casos, e principalmente, de média e alta complexidade. Esta dificuldade de acesso diz respeito a aspectos objetivos como a distância geográfica e os efeitos climáticos, ou seja, o atendimento aos nacionais se impossibilita devido a estes aspectos, sendo que do outro lado da fronteira, os recursos estão disponíveis.

Particularizando as desigualdades territoriais para cada experiência estudada, verifica-se que entre Santana do Livramento-Brasil e Rivera-Uruguai, o polo irradiador da demanda de serviços de média e alta complexidade foi a cidade brasileira, com um sistema de saúde de menor capacidade instalada, contrariamente à Rivera. Como Santana do Livramento se situa a aproximadamente $495 \mathrm{~km}$ de Porto Alegre, capital do Estado, e Rivera é uma continuidade geográfica, a solução mais favorável para à população seria mesmo uma articulação entre os sistemas. Esta situação se repõe em outras fronteiras, pois há uma tendência, no Brasil, das cidades principais se localizam mais próximas ao litoral.

A situação se inverte na tríplice fronteira, pois Foz do Iguaçu detém maior capacidade instalada de recursos de saúde do que Ciudad del Leste, distante $325 \mathrm{~km}$ de Assunção, capital do país.

No caso da fronteira entre Puigcerdá (Espanha) e Bourg Madame (França), a 
dificuldade de acesso à recursos no país ocorre não pela distância da cidade mais próxima, mas pela interrupção do tráfego devido a nevascas, sendo que há continuidade geográfica com a congênere espanhola. Como Puigcerdá detém maior capacidade de recursos instalada, incluindo um hospital, o polo irradiador foi Bourg Madame.

Entre Badajóz (Espanha) e Elvas (Portugal), o início da cooperação se deve ao fechamento da maternidade em Elvas e o atendimento tanto pré-natal como ao parto, deveria ser realizado em Évora, sita a 86 km, contrariamente à Badajoz, distante 16 $\mathrm{km}$.

Destacam-se, em todas as experiências, o protagonismo dos profissionais e gestores dos sistemas locais de saúde na articulação de estratégias para fomentar a cooperação entre os sistemas de saúde. A iniciativa sempre parte do profissional ou gestor com capacidade de liderança, que organiza diretamente ou utiliza outros meios para impulsionar o processo inicial.

Em Santana do Livramento e Rivera, é importante colocar que as iniciativas de integração e cooperação em saúde na fronteira Santana do Livramento-Rivera partem da Comissão Binacional Assessora de Saúde na fronteira Brasil-Uruguai, instituída em 2003. Esta Comissão propõe a realização de uma Conferência de Saúde, organizada pelos gestores dos dois municípios, onde se deliberou que deveria se constituir um Comitê Binacional de Integração em Saúde Integrada por profissionais e representantes institucionais relacionados com o campo da saúde, para estudar uma forma de articular as ações e serviços de saúde.

$\mathrm{Na}$ tríplice fronteira, há uma situação peculiar, pois a iniciativa parte de lideranças não integrantes do sistema local de saúde, mas protagonizada por funcionários da Empresa Pública Itaipu Binacional. Iniciada e monitorada pelo Grupo de Trabalho para Integração das Ações de Saúde na Área de Influência da Itaipu - GT Itaipu Saúde, o qual foi instituído, em 2003. É formalmente constituído por representantes dos governos locais, regionais e nacionais da saúde do Brasil e do Paraguai, os quais, entretanto, não tem presença assídua no GT. Não tem caráter executivo, unicamente consultivo. O objetivo do GT Itaipu Saúde é melhorar, com apoio da Empresa, a qualidade das ações de saúde na fronteira mediante planejamento e execução de atividades conjuntas prioritárias das políticas nacionais de saúde, com atenção para especificidades regionais. O fomento à ações integradas 
de saúde vem favorecendo uma articulação entre os gestores, na medida em que são acionados para implementar determinadas medidas. Não se identifica um continuum em suas ações, não apontando para expansão da harmonização de serviços e ações de forma mais consistente e sistemática.

Em Puigcerdá/Espanha e Bourg-Madame/França, na região dos Pirineus Orientais, a cooperação entre os serviços de saúde foi iniciada nos anos 2000, por iniciativa do gestor do sistema de saúde de Puigcerdá. Assim, desde esta época, por um acordo tácito, inicialmente as parturientes foram sendo encaminhadas ao hospital do sistema de saúde espanhol e os bebês registrados na cidade francesa. Justifica-se serem as parturientes as primeiras a serem atendidas, pois a região é sujeita a tempestades de neve com frequentes bloqueios das estradas aos centros maiores. A inexistência de equipamento na cidade francesa para atendimento dos partos favoreceu e facilitou o acordo. Em seguida foram ampliando a atenção sanitária para outros pacientes. Esta experiência foi indicada como referência de iniciativa exitosa por um dos integrantes da Comissão Executiva do Conselho Geral do Instituto Catalão de Saúde (Pérez, Nogueira, 2008).

Entre Badajoz e Elvas, os médicos dos hospitais locais foram os protagonistas do processo de cooperação em dois serviços de saúde - atendimento às parturientes e telemedicina. No primeiro caso, o fechamento da maternidade de Elvas, relatado anteriormente, leva a que o médico responsável pela mesma articule com a maternidade de Badajoz, inicialmente o atendimento ao parto e posteriormente se estende para pré-natal. Em relação à telemedicina, dois médicos dos dois municípios apresentam um projeto ao Fundo de Regionalização da União Européia e obtiveram o financiamento para equipar o hospital de Badajoz com um aparelho para tomografia axial computadorizada (TAC).

No caso de Santana de Livramento e Rivera, Puigcerdá e Bourg Madame e Elvas e Badajoz, as iniciativas foram facilitadas pelas necessidades territoriais locais e, na Europa, também os interesses por parte da União Europeia, em gerar uma interrede das regiões transfronteiriças com o objetivo de incrementar o nível de saúde das populações residentes na área. Contrariamente, na América Latina, as experiências se situaram à margem das instâncias do Mercosul e das autoridades ministeriais, embora contassem posteriormente com sua aceitação.

Ouro aspecto que favoreceu os processos de cooperação estudados foi o fato 
de serem iniciativas que partem de um ente público, tanto na prestação e financiamento dos serviços. Os obstáculos se situam no campo burocrático e na excessiva formalização para atuação interfronteiras. Observa-se que os aspectos formais, na área da fronteira, são encaminhados como nos demais municípios, sendo que o espaço territorial é, no campo legal e administrativo, tratado como limite e não como zona de transição.

Embora as distinções entre sistemas de saúde sejam reais, a sua operacionalização nas instâncias locais, por força da porosidade fronteiriça e a percepção do atendimento de necessidades concretas no campo da saúde, contribuem para a aceitação das inovações e resistências como o recorrente receio do estrangeiro, do diferente. No âmbito da pesquisa podem-se tirar as seguintes conclusões:

- As experiências reconhecidas de cooperação entre os sistemas de saúde decorrem de iniciativa dos gestores e atores políticos locais, pressionando os níveis superiores para reconhecimento e legalização dos acordos ou para resolver situações que fogem à sua alçada, como por exemplo, os registros de nascimento e falecimento. Ou formas de transferências monetárias para custeio dos serviços e protocolos de atenção à saúde.

- A demanda e a necessidade estão vinculadas ao acesso aos bens e serviços de saúde, seja por fatores climáticos, econômicos, debilidade do sistema instalado (baixa resolutividade, inexistência de equipamentos e pessoal técnico);

- Na fronteira brasileira, com exceção de Santana do Livramento, os arranjos e acordos informais não têm continuidade e dependem dos atores políticos ocupantes dos cargos decisórios em nível local, não favorecendo a criação de padrões institucionais para viabilizar sua sedimentação para além da vontade política do gestor;

- Os padrões de interação entre os países e entre as fronteiras determinam o maior ou menor interesse em articular ações e iniciativas de cooperação, sendo as cidades fronteiriças distintas entre si nos dois blocos econômicos, inviabilizando soluções globais que não decorram de propostas locais.

Ainda pode-se alertar, nas fronteiras brasileiras, para o papel desempenhado pelos Comitês de Fronteira e Comitês de Saúde enquanto atores importantes para mobilizar as forças políticas locais em direção a cooperação de sistemas e ações e 
serviços de saúde.

Há, no entanto, a necessidade de ampliar o espectro político para além do partidário e obter uma expertise para manejo dos recursos governamentais disponíveis, pois os discursos dos atores não tocam em possibilidade de usar, por exemplo, os recursos do Programa de Desenvolvimento da Faixa de Fronteira, do Ministério da Integração Nacional com recursos do Plano de Aceleração do Crescimento (Brasil, 2011).

Nas fronteiras européias destaca-se, nas iniciativas locais, a capacidade de incorporar os fundos estruturais da União Européia em benefício da cooperação em nível local, o que é favorecido pelo estilo de governabilidade dos países envolvidos, com democracias consolidadas onde há o respeito às normas e regulações legais em qualquer um dos planos hierárquicos.

Não se pode olvidar que todas as experiências se iniciaram em um tempo de reajustes estruturais das economias para fazer frente à crise capitalista a partir dos anos 1980. Nos dois últimos anos houve o aguçamento da crise em Portugal e Espanha e uma relativa potencia do Brasil em fazer frente à mesma. Como o futuro das experiências depende em parte dos resultados das ações e medidas nacionais e globais, a tendência é um retraimento das ações cooperativas e mais um retorno a marca da fronteira como um limite nacional.

Não se buscou estabelecer, a partir da análise das experiências, receitas ou modelagem para cooperação entre sistemas de saúde de países distintos. Pretendeuse, sim, no campo da gestão, colaborar para fortalecer o programa em curso - SIS Fronteiras, desenvolvido pelo Ministério da Saúde, Secretarias Estaduais e Municipais de Saúde. E, ainda, otimizar os recursos financeiros alocados em municípios brasileiros de fronteira através dos protocolos derivados do Pacto pela Saúde, garantindo atenção integral também aos não nacionais, como um direito definido pelo Pacto dos Direitos Econômicos Sociais e Culturais sob a chancela da ONU e do Parlamento Brasileiro.

\section{Referências}

ASTORGA J., Ignácio. Estudo da Rede de Serviços de Saúde na Região de Fronteira Argentina, Brasil e Paraguai: 2001-2002. Brasília: Organização Pan-Americana da Saúde, 2004. 
BRASIL. Ministério da Integração Nacional. Bases de uma política integrada de desenvolvimento regional para a faixa de fronteira. Secretaria de Programas Regionais do Ministério da Integração Nacional. Programa de Desenvolvimento Social da Faixa de Fronteira. Brasília, 2004. [data da consulta: 09 maio 2006] Disponível em: $<$ http://www.igeo.ufrj.br>.

BRASIL. Ministério da Integração Nacional. Programa de Desenvolvimento de Faixa de Fronteira. Brasília, Secretaria de Programas Regionais do Ministério da Integração Nacional, 2009. [data da consulta: 25 ago. 2011] Disponível em $<$ http://www.integracao.gov.br/programas/programasregionais/fronteira.asp?area=spr fronteira>.

D.G. FONDOS COMUNITARIOS ESPAÑA/D.G. DE DESARROLLO REGIONAL PORTUGAL. Programa operativo de cooperacion transfronteriza España-Portugal 2007-2013. [data da consulta: 15 ago. 2011] Disponível em: < http://www.integracao.gov.br/c/document_library/get_file?uuid=e5ba704f-5000-43dfbc8e-01df0055e632\&groupld=10157 >.

DAIN, Sulamis. Subsídios para a formulação de um Sistema de Integração dos Modelos de Prestação de Serviços de Saúde no MERCOSUL e nas Regiões de Fronteira: Harmonização e Transfronteirização dos Sistemas de Saúde. Em: GALLO, Edmundo e COSTA, Laís (Orgs). SIS-MERCOSUL: uma agenda para a integração. Brasília: OPAS/Ministério da Saúde. 2004.

GALLO, Edmundo. SIS-Mercosul: Uma agenda para a Integração. Em: GALLO, Edmundo e COSTA, Laís (Orgs.). SIS-MERCOSUL: uma agenda para a integração. Brasília: OPAS/Ministério da Saúde. 2004.

GIOVANELLA, Ligia et al. Saúde nas fronteiras: acesso e demandas de estrangeiros e brasileiros não residentes ao SUS nas cidades de fronteira com países do MERCOSUL na perspectiva dos secretários municipais de saúde. Cadernos de Saúde Pública, Rio de Janeiro, 2006. [data da consulta: 16 mar. 2009] Disponível em: <http://www.scielo.br/scielo.php?script=sci_arttext\&pid=S0102-

$311 \mathrm{X} 2007001400014 \&$ lng $=$ pt\&nrm=iso $>$.

GUIMARÃES L, GIOVANELLA L. Integração Europeia e Políticas de Saúde: repercussões do mercado interno europeu no acesso aos serviços de saúde. Cadernos de Saúde Pública, 22(9): 1795-1808, 2006.

GUIMARÃES L, GIOVANELLA L. Processo de integração regional no Mercosul e as repercussões nos sistemas de saúde de cidades fronteiriças. Anales del $X$ Congreso Internacional del CLAD sobre la Reforma del Estado y de la Administración Pública. Santiago, Chile, 18-21 oct. 2005 a.

GUIMARÃES, L., QUEIROZ, W. Integração Européia e Acordos Fronteiriços em Saúde na Euroregião de Extremadura-Alentejo. Integração regional e políticas de saúde: União Européia e MERCOSUL. Tese (Doutorado) Saúde Pública da Escola Nacional de Saúde Pública Sérgio Arouca, da Fundação Oswaldo Cruz do Ministério da Saúde do Brasil (Ensp/Fiocruz/MS). 2007.

NOGUEIRA, Vera Maria Ribeiro; DAL PRA, Keli Regina e FERMIANO, Sabrina. A diversidade ética e política na garantia e fruição do direito à saúde nos municípios brasileiros da linha da fronteira do MERCOSUL. Cadernos de Saúde Pública [online]. 23(2):227-236, 2007 [citado 2009-03-16]. [data da consulta: 12 mar. 2011] Disponível 
em:

$<$ http://www.scielo.br/scielo.php?script=sci_arttext\&pid=S0102-

311 X2007001400012\&lng=pt\&nrm=iso $>$.

NOGUEIRA, Vera Maria Ribeiro; DAL PRÁ, Keli. MERCOSUL: expressões das desigualdades em saúde na linha da fronteira. Ser Social (UnB), Brasília, (18):159188, 2006.

NOGUEIRA, Vera Maria Ribeiro; PEREZ, Roser G;. La construcción de los derechos sociales y los sistemas sanitarios: los desafíos de las fronteras. Katalysis, 12(1):5058, 2009.

SIMIONATTO, Ivete; NOGUEIRA, Vera M.R. ; GOMEZ, M. B. Aspectos legais do Direito à Saúde. Em: SIMIONATTO, Ivete; NOGUEIRA, Vera M.R. (Org.). Dilemas do Mercosul: reforma do Estado, Direito à Saúde e Perspectivas da Agenda Social. Florianópolis: Lagoa Editora, 2004. p. 81- 94. 